\title{
A METHOD FOR STUDYING VIBRATIONS TRANSMITTED TO THE HANDS
}

\author{
BY
}

\author{
JOHN N. AGATE and H. A. DRUETT
}

From the Department for Research in Industrial Medicine (Medical Research Council), The London Hospital.

There is an increasing tendency in engineering practice to put into the hands of workers powerdriven tools of all kinds for work which involves hammering, chiselling, grinding and polishing. The speed and efficiency of working is increased and the physical labour involved is lessened, but most of these portable tools cause vibration to be transmitted direct to the hands. Studies completed in the past 30 years, and those now in progress, attribute clinical effects to the use of such tools, and the factor common to them all is the vibration set up. Middleton (1930) reported the Raynaud phenomenon in those who operated pounding-up machines in the boot and shoe trade, but these machines have since passed out of use. The same syndrome, variously called 'white fingers,' ' dead fingers,' or ' dead hand,' has since been described frequently in the literature. Maria Seyring (1930) reported it in those who were fettling iron castings with pneumatic tools. Hunt (1936) also observing pneumatic tool workers, pointed out that the hand which was worst involved was the one which appeared to be subjected to the most vibration, that is, the hand in front which grasps the tool at its striking end. Other cases were recorded by Teleky (1927), McLaren (1937), Bennett, Waine and Bauer (1942), Barker and Hines (1944), Lindquist and Flemberg (1945), and Hunter, McLaughlin and Perry (1945). The latter studied 286 men who had worked with pneumatic tools of various sizes, and in a variety of occupations. White fingers were noted in 161 , and developed usually after 2-5 years of such work. As in all the foregoing examples cold was the principal precipitating factor. In 148 cases in this series there were small circumscribed areas of decalcification demonstrable in radiographs of the carpal bones, a fact which had previously been noted by Brailsford (1934) (1936) and McLaren (1937), though the appearance is certainly not to be found solely in pneumatic tool workers. The incidence of arthritis of the elbow and wrist joints and of injury to the ulnar nerve or fasciae of the palm was not high in this series, though there have been references to these conditions since 1930.

In the war years there has been a rapid increase of work involving the polishing of steel castings and scurfing of aluminium alloy castings used in the manufacture of aero engines. The practice favoured at the time of writing is for a hand tool to be used to facilitate work in awkward corners

$\mathbf{N}$ and within the castings. The tool consists of a hand grip attached to a flexible cable drive running from an electric motor suspended from the roof. Various polishing, grinding and cutting heads can be used in this tool, and further cases of Raynaud's phenomenon have resulted, due to the intense vibration set up in the hands (Telford, McCann and MacCormack (1945)). Other cases are known to be occurring in industries where metal components are held in the fingers for grinding against grindingwheels on fixed mountings, the vibration in this case being transmitted not from the machine but from the article being ground.

It has been suggested that various other factors assist the appearance of clinical effects from the use of vibrating tools. The current of cold exhaust air from pneumatic hammers is one, the hardness of the material on which the men use their tools is another. But the rates of rotation of the grinding tools or of the hammer strokes in pneumatic tools have been particularly considered. Cummings (1940) and Mills (1942) suggest that hammer strokes of 2500 per minute are more likely to cause Raynaud's phenomenon than other speeds, and in the cases of Hunter, McLaughlin and Perry a higher incidence was noted in those who worked with tools at 2000 to 3000 strokes per minute than at speeds above or below. The issue is, however, complicated by the fact that on the softer metals such as hot rivet heads, a slower type of hammer is used, and the decreased incidence of the phenomenon might be from either cause. The heaviest pneumatic tools, besides being the slowest, are usually used pointing downwards, and the work is done as much by the tools' weight as by the efforts of the workman. Similarly, the fastest tools are used on the lightest jobs, so that the grip and exertions of the operator can be less, and he probably absorbs less vibration in consequence. Rotating polishing tools are usually used at about 1900-4000 r.p.m., but light compressed air turbine tools for fine work may work at above 30,000 r.p.m., and in these the vibrations set up are apparently of a finer type, and the operator's grip on the tool is light.

No great advance towards discovering the cause of the clinical effects of vibrations, or of obviating them, is likely to be possible until a method for accurately recording their nature has been evolved. When this is done, it might be possible by correlating clinical and physical data to determine whether there is a critical frequency likely to cause the 
syndrome; secondly, it might be possible to predict the chances of a given tool producing it, and thirdly, the efficacy of countermeasures such as the use of absorbent hand grips and pads could be studied. But the vibration set up by any of these tools is not of a single frequency nor has it a curve of simple sine-wave type. There may be a fundamental of low frequency, but, as in the case of a musical instrument, there are harmonics of higher frequency overlying it. In a rotating machine the fundamental bears a relation to the shaft speed, and the harmonics which modify it are provided by the irregularities of the shaft and its bearings and by such factors as the number of teeth on the cutting head, while in an oscillating pneumatic hammer the fundamental relates to the to-and-fro action of the internal piston, though the natural resonance of the metal of which it is made sets up a secondary set of high-frequency vibrations. In both types the natural resonance of the materials worked upon is transmitted back into the tool and thence to the workman's hands, while the power of his grip and the weight of his arms modify the vibrations. What is more, the vibration of the tool while idling differs from that while it is working, and the frequencies alter from time to time as the man presses harder to grind and so slows down his machine.

\section{Fourier Analysis}

When harmonics are superimposed on a fundamental vibration they modify its waveform, as depicted graphically, from the simple sine-wave curve. In the vibrations of machinery, even more than in simple instrumental notes the waveform becomes exceedingly complex. Fourier at the beginning of the last century showed that any uniformly repetitive phenomenon, however complicated its waveform, could be resolved as the sum of a series of waves of the sine type, the frequencies of the component waves being simple multiples of that of the main wave under consideration. These higher frequency waves are its harmonics. In the present investigation an electrical Fourier analysis has been made on the composite vibrations, to break them up into their constituent harmonics and determine the frequency and amplitude of each.

If it can be assumed that the local pathological effects of vibrations in the hands are related in some way to the quantity of energy absorbed by the tissues, it is unreasonable to assume that the effects of energy represented by the low-frequency components are necessarily the same as those represented by the high frequency. Already there are being noted bizarre biological effects from very high-frequency vibrations outside the audible range, otherwise known as 'supersonic' vibrations. In the same way the biological effects of high-frequency alternating currents differ widely from those of low frequency. It is by no means proven, though it is likely, that the pathological process set in action by the vibrations takes place entirely locally in the palm of the hand or the digits; arteriograms have shown spasm occuring locally in the peripheral vessels in the hand, and this may be the case in other examples of Raynaud's phenomenon of different aetiology. The question will probably remain unsolved until there is histological evidence taken from the vessels in the hand of a pneumatic tool worker. The opportunities for such a study are naturally very infrequent.

The requirements of any method for studying the problem experimentally are therefore that it shall make possible the analysis of the total vibration taking place at any given moment in terms of amplitudes and frequencies, which may lie between 20 and 10,000 vibrations per second or above. The time taken over each analysis must be short or the conditions of the experiment may have changed meanwhile. The apparatus must be sufficiently portable to enable it to be taken to the worker at his job, for most of the tools and materials worked upon are too bulky to be studied in the laboratory. Whatever the method of picking up the vibrations, it must not hinder the use of the workman's hands in any position, nor must his normal grip be materially altered. Mechanical or fluid transmission from the vibrating surfaces to any recording apparatus is clearly useless both because of the inertia of any such system, and because of its impracticability on all of the above counts. An electrical method has therefore suggested itself.

\section{The Piezoelectric Crystal}

Certain crystals which have no centre of symmetry, such as quartz, tourmaline, or Rochelle salt, exhibit piezoelectric effect, that is, when distorted by pressure they develop an electric potential, and, as discovered by the Curies, the potential generated is proportional to the pressure applied. A crystal suitably mounted can equally well be distorted by being shaken, and therefore can be used to pick up vibrations. Rochelle salt (sodium potassium tartrate) crystals have 1000 times the piezoelectric effect of quartz, and can be grown artificially. Variations in the response of individual crystals, particularly in response to changes of temperature, are minimized by making laminated or 'bimorphic' crystals of two plates bonded together. Such crystals will accurately follow vibrations at least up to 10,000 cycles, and their electrical response can be analysed by methods to be described below. The crystal pickups used in our experiments were of Rochelle salt and were supplied by Messrs. Rothermel, Ltd. They were fixed in mountings measuring $25 \times 32 \times 4 \mathrm{~mm}$. The crystal projected out of one end, and was otherwise covered by a metal housing; at the other end was fixed the screened lead that conducts the response. In the early stages the crystal was strapped directly to the tool under investigation, but it was found to become damaged by handling and pressure, and in particular by the heat generated during certain of the grinding work, for the piezoelectric properties of crystals are permanently lost after exposure to $130^{\circ} \mathrm{F}$. It was therefore necessary to make a 


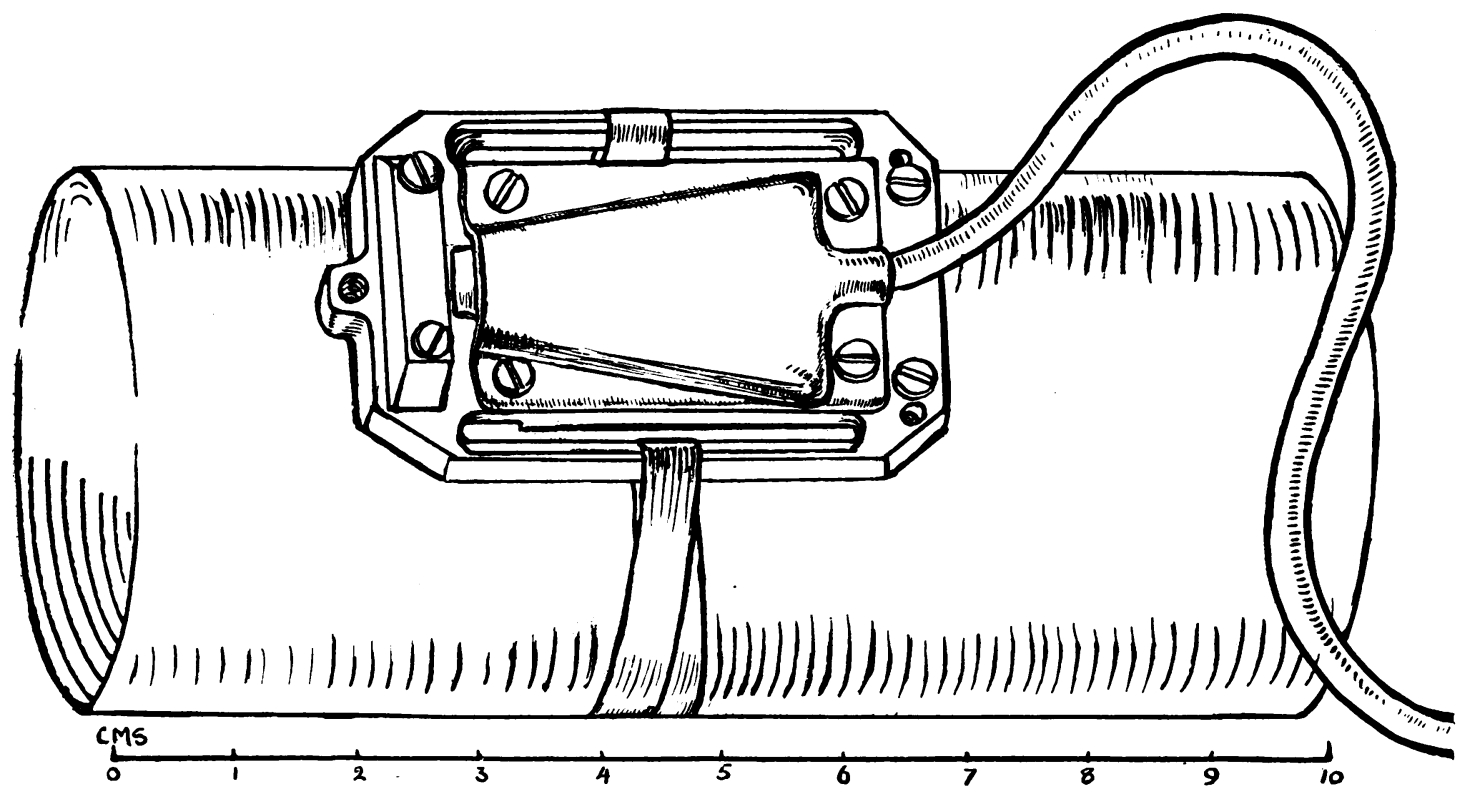

FIG. 1.-The piezoelectric crystal and mounting, shown strapped to a cylinder of metal. The pointed setscrews which make direct contact are hidden.

new mounting. The crystal in its original cover was screwed to a metal base-plate (fig. 1) to reduce the pressure distortion and the risk of damage by heat, the only disadvantage being a slight increase in bulk, though the pickup would still lie comfortably in the palm of the hand. The base-plate had a protective bar at one end to guard the naked point of the crystal and a slot along each side by which it could be tightly bound by inelastic tapes to the handle of any tool. Direct metallic contact was ensured by four pointed and adjustable steel set-screws which allowed the pickup to rest on a surface of any curvature, and which could be withdrawn if readings were to be taken from skin or any other soft surface. As a rule the pickup was attached to the handle of a tool at the point where it is usually held, and the man's hand would lie on top of it, the disturbance to his normal grip being negligible and the limitation of his movements solely that imposed by the length of the pickup lead. Thic base-plate was designed also so that it could be clamped when desired to one arm of an electrically maintained tuning fork. The amplitude of the vibration at the point of clamping was measured optically with a microscope having a micrometer eye-piece calibrated against a stage micrometer, a point on the crystal itself being observed under horizontal illumination as it moved to and fro. The response of the pickup to a standard deflection could by this means be checked from time to time: this was found particularly necessary as the crystal had to be subjected to rough handling during normal working conditions.

\section{Analysis of the Pickup Response}

In theory the voltage generated in the crystal pickup could be made to displace the electron beam of a cathode-ray oscillograph and a picture of the waveform would appear on the fluorescent screen. From this the constituent harmonics might be calculated. In practice, while this picture is relatively easy to obtain, its interpretation in terms of the harmonics is at best difficult and inaccurate. Usually it is impossible. The transience and the variable nature of the vibrations from machines do not simplify the matter.

To avoid the difficulties use has to be made of the properties of electrical filter circuits through which the pickup response can be passed. A ' high-pass filter' will entirely reject frequencies below a certain chosen value and pass those above. A 'low-pass filter' will pass frequencies below a certain value and reject those above. A 'band-pass filter' will pass frequencies between two chosen values and reject those above and below. This band in the latter type can be made almost narrow enough to select a single frequency alone, and this principle is embodied in the instrument first used in these experiments, a Harmonic Wave Analyser to the specification of Messrs. Marconi Instruments, Ltd. This apparatus was a battery model and portable, besides being sensitive and capable of defining frequencies within narrow limits. It could be tuned on a graduated dial to any frequency in the range under investigation, and the proportion of the crystal response corresponding to that frequency measured on a millivoltmeter. In operating the instrument the tuning dial was turned slowly and the points of maximum deflection of the meter needle were observed and the corresponding reading in cycles per second on the dial noted. The meter registered throughout, and the 'peaks' of its deflection had to be determined to place the principal frequencies. Graphs could then be constructed 
by plotting output in millivolts and frequency as ordinate and abscissa respectively. The readings taken by this means, though laborious, were accurate to within a few cycles per second and gave results that could be reproduced provided that the vibration remained constant throughout the period of the observation. The method was therefore applicable to a tool that was idling at constant speed, or to any tool in which the rotation speed was not dependent on the load applied to the cutting face. In practice it was found of little use with any machine under load because the small variations of speed from second to second made the 'peaks' on the meter impossible to follow. As a full set of readings might take up to 30 minutes the instrument could not be used to record the vibrations during a short operation such as striking a few blows with a pneumatic hammer, or a few seconds' grinding of an iron component against a wheel. A method giving instantaneous readings had to be sought.

\section{The Acoustic Spectrometer}

In the later stages an Acoustic Spectrometer, made by Messrs. Standard Telephones and Cables, Ltd., was used (fig. 2). In principle this is a highspeed waveform analyser, giving a visual indication in the form of a spectrum of the individual components of a complex wave. A block diagram of the electronic circuits is shown (fig. 3). The incoming signal from the pickup is first amplified, provision being made to vary the degree of amplification (the ' gain' control), and it is then applied to 27 bandpass filters of the wide type already described, arranged in parallel. The filters are designed to operate over an interval of $1 / 3$ rd of an octave each, and are constructed in such a way that if a signal is received in the middle of one pass range, the adjacent filters will pass only 20 per cent. of the actual amplitude. The frequency of the middle of the lowest band is 40 cycles per second, and that of the highest 16,000 per second. The output of each of the filter circuits is rectified and stored in condensers. In turn the output of the condensers is injected through a motor-driven rotating switch into a ring modulator and converted into alternating voltages of 3000 cycles per second. The output of the modulator is passed through a band pass filter to suppress harmonics and after further amplification and rectification is applied to the $\mathrm{Y}$ plates (vertical deflection) of the cathode ray tube, where a vertical band of fluorescence appears above a base line. The rotating switch makes contact with one of a series of constant potentials at the same time as it is applying the output of the storage condensers to the modulator. These fixed potentials are applied to the $\mathrm{X}$ plates (horizontal deflection) at the same time as the $Y$ deflection, with the result that 27 separate spots or vertical lines appear on the screen (fig. 4). The height of each line is proportional to the vector sum of the amplitudes of the harmonic

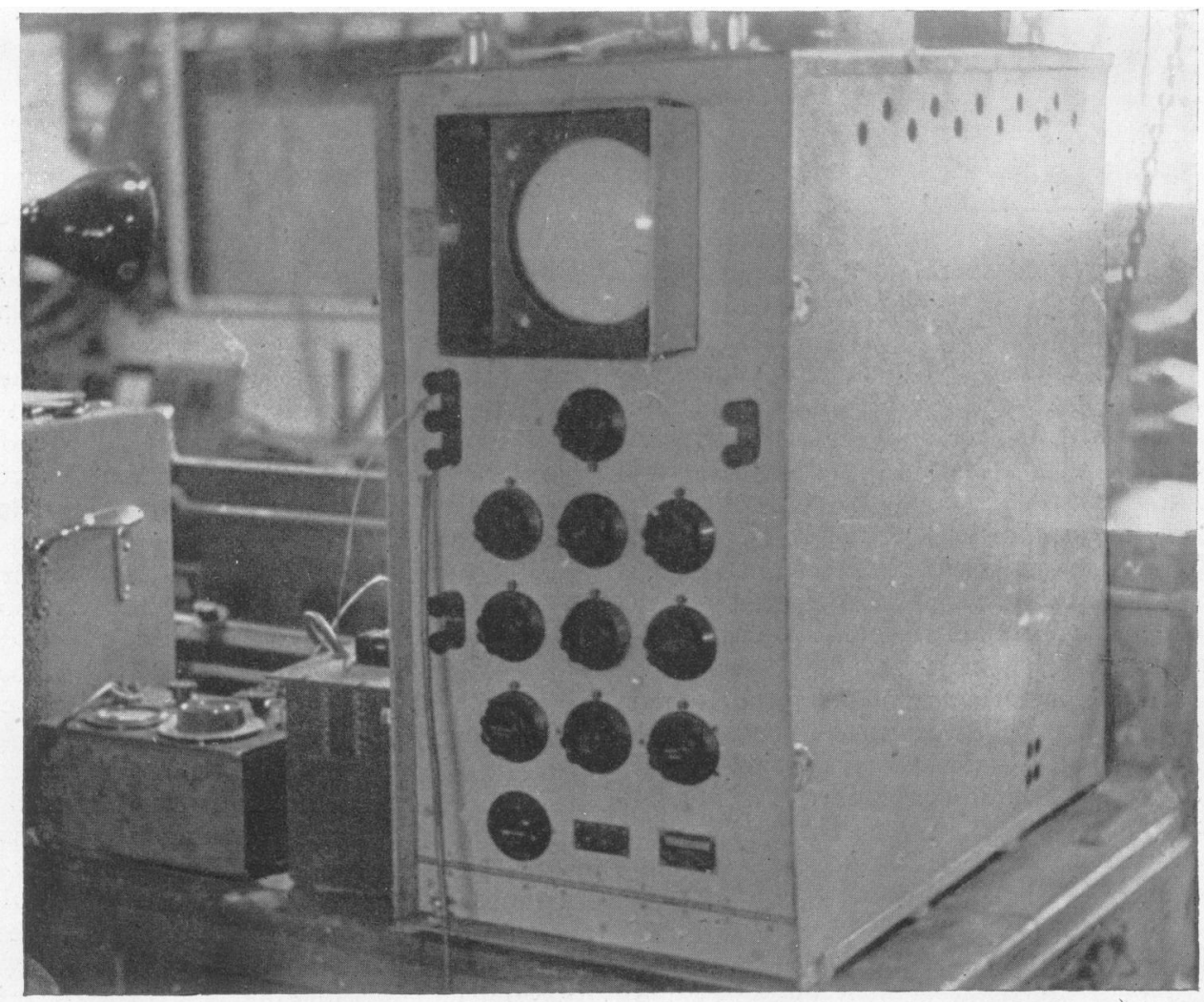

FIG. 2.-The Acoustic Spectrometer, with camera and hood removed. 


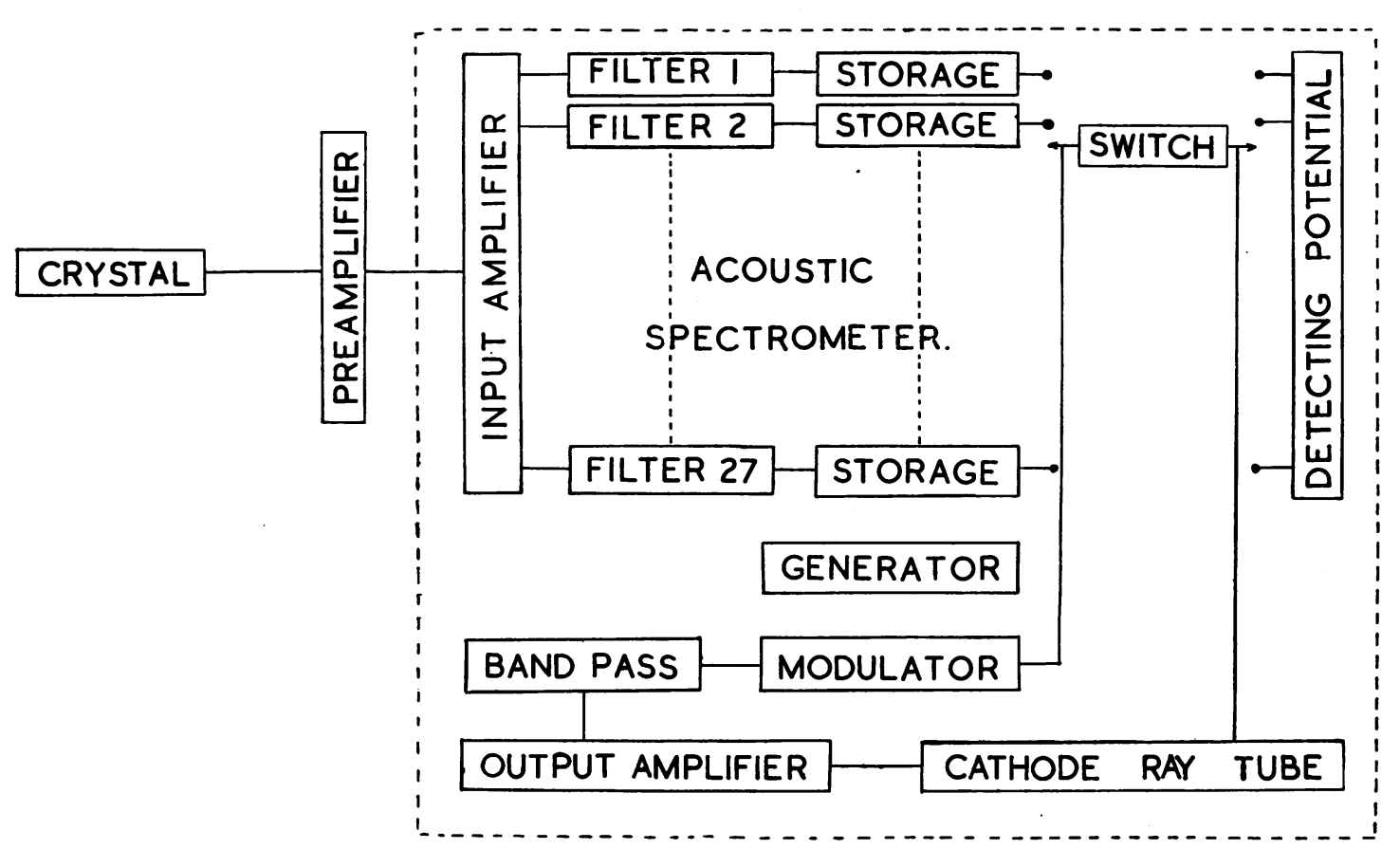

FIG. 3.-Block diagram of the electronic circuits in the Acoustic Spectrometer.

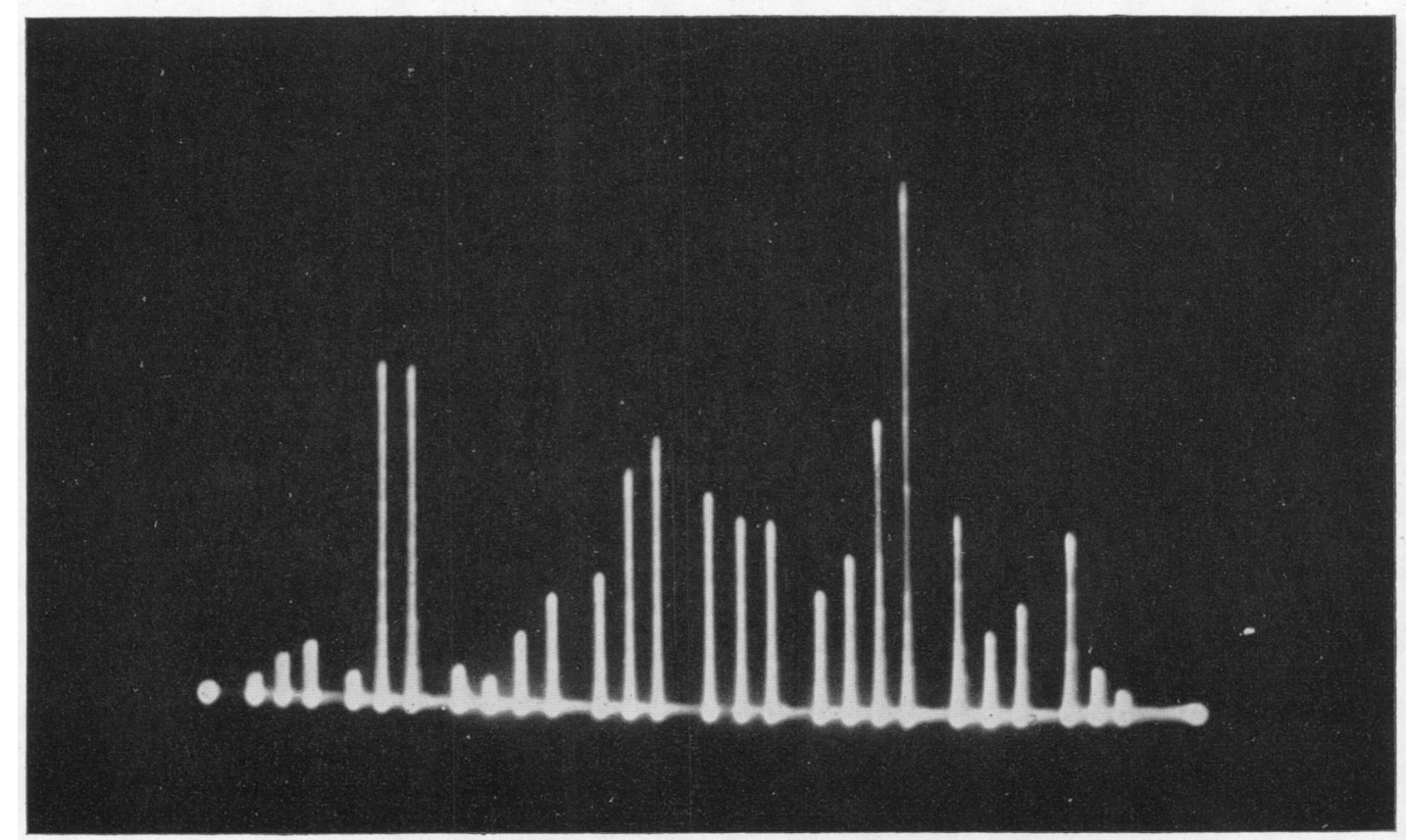

Fig. 4.-Fhotograph of the screen of the Acoustic Spectrometer taken while recording. 
in the band it represents, and it can be measured by dividers, or alternatively the whole image can be photographed and measurements taken at leisure. The switch rotates at over 20 times per second, and due to this and the afterglow of the cathoderay tube a continuous picture is obtained. The response of the filters is so arranged that, for an equal voltage of input, lines of equal length are drawn with an error of less than 10 per cent. A switch is incorporated whereby the charge and discharge times of the storage condensers can be altered. In the 'short' position the charging time is approximately 20 milliseconds, and that of the discharge is about 100 milliseconds. In the ' long' position these values are increased by a factor of 10 .

To give a greater range of permissible input voltages, the crystal output was first applied to a separate amplifier whose characteristics were known to be linear in the range of frequencies with which this investigation is concerned. The gain stages of this amplifier were $-20,0,20,40$, and 55 decibels. In addition to this the preamplifier had a much higher input impedance than that of the acoustic spectrometer, and was therefore well suited to receiving the crystal output.

The advantages of this instrument over the Harmonic Wave Analyser are that the whole of the vibration spectrum at any given moment can be seen at once and instantaneous records taken, so that many more readings are possible in a short time, and mean values can be assessed for any period in which much variation is taking place, by taking several records rapidly. The disadvantages are its bulk, the fact that it requires alternating mains current, and individual frequencies cannot be more accurately localized than within the filterband limits already prescribed for the instrument. This makes it necessary to assume, until it is proved to the contrary, that the damaging frequencies will lie between certain narrowly defined limits but need not be individually determined with great accuracy; to assume the converse is to imply that all biological tissues are identical in behaviour. Small variations in the peaks due to the speed of the tool varying under load do not as a rule affect the response within a band, and this is advantageous.

Provision is made for the use of a cinematograph camera with the spectrograph, but it was found that the image on the screen varied very little from moment to moment when the tools were being worked, and not at all when they were idling. It was decided to use a $35-\mathrm{mm}$. miniature camera and to take individual exposures, one only for an idling tool, and three, for the calculation of mean values, for a tool under the variations of load. The camera was a Zeiss 'Contax' with an f.2 Sonnar lens, mounted at $26.3 \mathrm{~cm}$. from the cathoderay tube screen in a detachable hooded mounting. To provide for the unusually short object-lens distance a tubular extension was made to fit between the detachable lens of the camera and its focal plane. The film used was Kodak 'Super XX' Panchromatic, and it was developed under standard conditions to minimize any error from the shrinkage of film. The exposure required at full aperture was $1 / 10$ th second; more than $1 / 5$ th second caused halation, and less than $1 / 10$ th caused some of the image to be lost as the electron beam swept from one side of the screen to the other, unless the shutter happened by chance to be synchronous with one completed sweep. The lengths of the lines on the negative film were later measured on a simple microscope with a 3-inch objective and a micrometer scale in the eye-piece.

The mid-frequencies of the band-pass filters in the spectrometer, expressed in cycles per second, were as follows :

TABLE 1

\begin{tabular}{r|r|r|r|r|r}
\hline 1 & 40 & 10 & 320 & 19 & 2500 \\
2 & 50 & 11 & 400 & 20 & 3200 \\
3 & 64 & 12 & 500 & 21 & 4000 \\
\hline 4 & 80 & 13 & 640 & 22 & 5000 \\
5 & 100 & 14 & 800 & 23 & 6400 \\
6 & 125 & 15 & 1000 & 24 & 8000 \\
\hline 7 & 160 & 16 & 1250 & 25 & 10,000 \\
8 & 200 & 17 & 1600 & 26 & 12,500 \\
9 & 250 & 18 & 2000 & 27 & 16,000 \\
\hline
\end{tabular}

\section{Calibration of the Spectrometer}

For the calibration of the preamplifier and the acoustic spectrometer the method used was to apply the output of a signal generator through a millivoltmeter to the input of the preamplifier. The response of the spectrometer was measured on the screen with a steel rule. The amplifications provided by the various settings of the preamplifier were checked with standard input voltages and were found to be correct. The 'gain' control of the spectrometer was in arbitrary divisions marked $0-130$, and the instrument was calibrated as follows. The signal generator was tuned in succession to the centre of each band, and the voltage necessary to trace a line of $4 \mathrm{~cm}$. on the screen was determined. In all cases it was found to be the same, within the limits given above. The signal generator was then tuned to one of three chosen frequencies, and, with the 'gain' knob set in each of nine selected positions $(130,120,110, \ldots 50)$ readings were taken of the applied voltage necessary to produce a line measuring $\frac{1}{2}, 1,2, \ldots 8 \mathrm{~cm}$. The mean of the readings for each of the three frequencies was calculated and plotted to give the family of curves shown in fig. 5. From this graph it is therefore possible to read off the millivolts input which corresponds to the length of the line appearing on the screen of the cathode-ray tube. A series of lines of standard length were produced and photographed. From this the ratio of the length of the image on the negative film to the length of the line on the screen was determined, and from this the alternative ordinate was inserted in fig. 5 , which enables the length of line on the negative film to be converted directly into terms of input millivolts. 


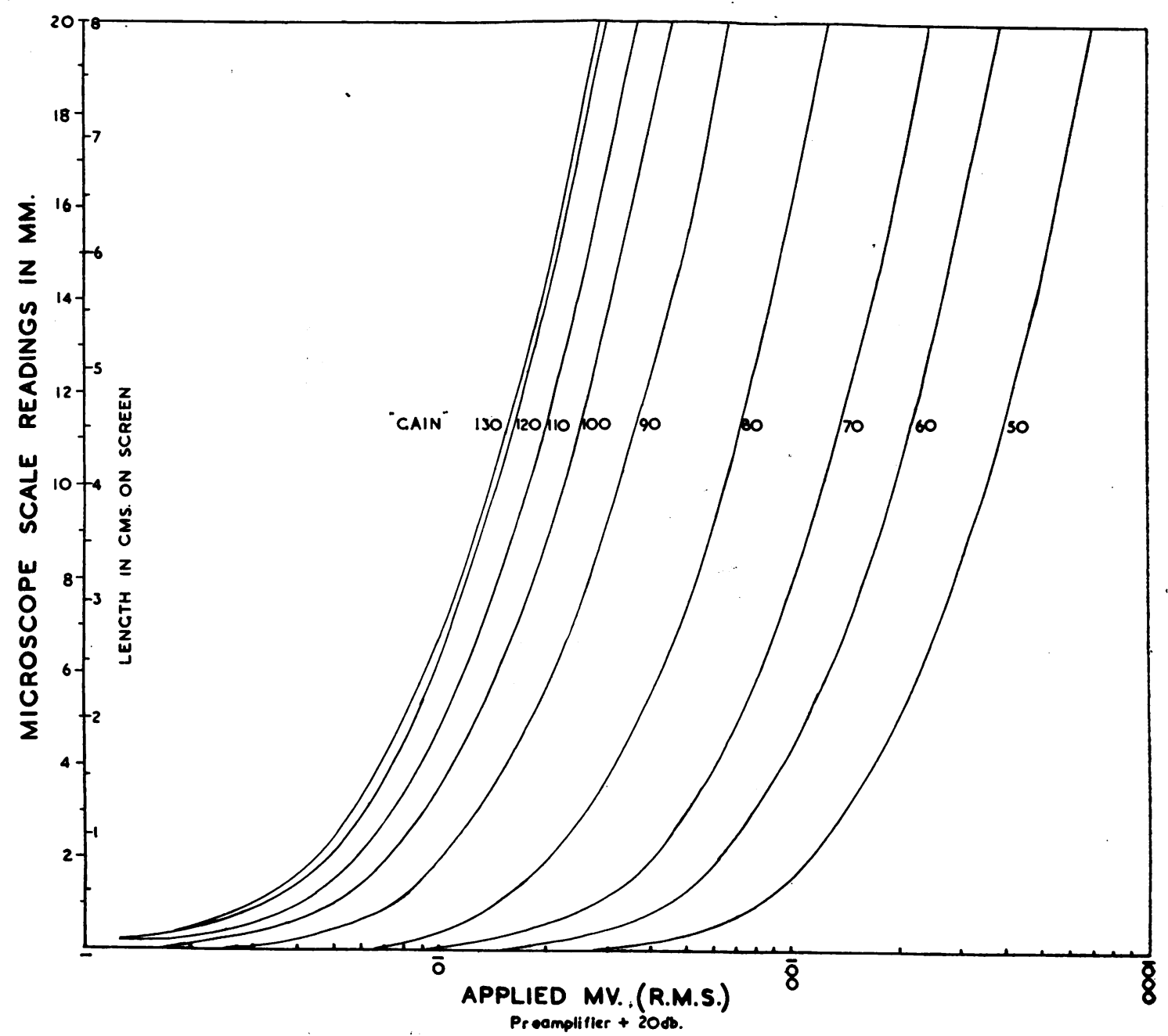

FIG. 5.-Graph for conversion of length of the lines on the screen into millivolts of input voltage. (For explanation, see text.)

The voltage generated by a piezo crystal is given by

$$
\mathbf{V}=\mathrm{KAF}^{2}
$$

where $\mathrm{V}$ is the voltage generated

$A$ is the amplitude of the vibration

$\mathrm{F}$ is the frequency of the vibration

$\mathrm{K}$ is a constant depending on the geometry and composition of the crystal.

The same voltage will therefore be generated by a crystal when subjected to a vibration of an amplitude of either $1 \mathrm{~mm}$. at 100 cycles per second, or $10 \mu$ at 1000 cycles per second, or $0 \cdot 1 \mu$ at 10,000 cycles per second.

It is necessary to bear this in mind always when viewing the whole spectrum on the cathode-ray tube screen, otherwise an erroneous impression will be gained because the higher frequencies will appear to have a much greater amplitude than is really the case after the correction has been applied. Similarly, a false impression would be obtained by measuring the total output of the crystal irrespective of the frequencies causing the generated voltage.
While it is not possible to deduce from the acoustic spectrometer records the amplitude corresponding to each individual frequency, a good approximation can be made by writing

$$
\mathrm{V}_{\mathrm{b}}=\mathrm{K} \cdot \mathrm{F}_{\mathrm{b}}{ }^{2} \cdot \mathrm{A}_{\mathrm{b}}
$$

where the suffix $b$ refers to the mid-frequency of the band-pass filter. If logarithms of both sides are taken the result is

$$
\log \mathrm{V}_{b}=\log \mathrm{KF}_{\mathrm{b}}{ }^{2}+\log \mathrm{A}_{\mathrm{b}},
$$

and it follows therefore that if $V_{b}$ is plotted against $A_{b}$ on log-by-log paper a series of parallel straight lines will be produced, all at 45 degrees to both axes, their position being determined by the value of $F$ chosen. In this manner a second graph can be constructed enabling readings of $\mathrm{V}$, the voltage generated as obtained from the first graph, to be converted directly into amplitudes, once the value of $\mathrm{K}$ is known. The makers of the crystals give data which leads to a value of

$$
\mathrm{K}=1.18 \times 10^{-5} \text {, }
$$

when $\mathrm{V}$ is expressed in millivolts and the amplitude 
in microns. This value for $\mathrm{K}$, which is based on data obtained at 1000 cycles per second, was checked by attaching the crystal to an electrically-maintained tuning fork suitably counterbalanced and running at a frequency of 100 cycles per second. The amplitude of the vibration was then determined microscopically as already described, and a value of $K$ was calculated and found to be substantially in agreement with that obtained from the data supplied by the makers.

\section{Specimen Result}

Under investigation was a portable grinding tool which consisted of a metal hand-piece through which passed a flexible cable transmitting power from an electric motor suspended from the roof. The hand-piece was held by the operator in both his hands, and fastened in it was a mounted silicon carbide grinding stone of $1 \frac{3}{4}$ inches diameter. The spindle of the stone was $\frac{1}{4}$ inch in diameter and was found to be $3 \frac{1}{2}$ thousandths of an inch out of true. The machine was rotating at 4050 r.p.m. as checked on a revolution counter, and the operator was grinding the smooth surface of a medium-sized hollow steel casting. The crystal mounting was strapped to the hand-piece directly under the palm of his left hand, which was placed in front. While he was working the response of the crystal was shown on the screen and produced a picture resembling that of fig. 4 . The height of the highest lines was adjusted by using the 'gain' control to approximately $5 \mathrm{~cm}$., and the position of this control noted. Three exposures were taken and the film processed. The lines reproduced on the negative film were measured and the mean value of the three taken. The lengths of the lines were converted into millivolts by the first graph (fig. 5), and thence into terms of amplitude in microns by using the second graph described above, and the results obtained were as follows :

TABLE 2

\begin{tabular}{c|c|c|c}
\hline $\begin{array}{c}\text { Frequency } \\
\text { Band } \\
\text { (cycles per } \\
\text { second) }\end{array}$ & Amplitude & $\begin{array}{c}\text { Frequency } \\
\text { Band } \\
\text { (microns) } \\
\text { (cycles per } \\
\text { second) }\end{array}$ & $\begin{array}{c}\text { Amplitude } \\
\text { (microns) }\end{array}$ \\
\hline 40 & 330 & 320 & $9 \cdot 7$ \\
50 & 240 & 400 & $2 \cdot 6$ \\
64 & 390 & 500 & $1 \cdot 05$ \\
80 & 185 & 640 & $0 \cdot 62$ \\
100 & 62 & 800 & trace \\
125 & 94 & 1000 & trace \\
160 & 47 & 1250 & nil. \\
200 & 37 & and up- & wards \\
250 & 24 & & \\
\hline
\end{tabular}

By using such series of results comparisons are possible to show the differences between the vibra- tions reaching the right and the left hands, or the effect of interposing an absorbent rubber surface between the handle of the tool and the man's palm. These will be made the subject of a further communication.

\section{Summary}

The lesions which result from the absorption of vibrations, particularly Raynaud's phenomenon occuring in the hands, and the various types of work which may cause them, are briefly discussed. The type of vibrations set up, their analysis, and the usefulness of piezoelectric crystals for picking them up experimentally. are considered. Two instruments for analysis of the response from the pickup crystals were used in experiments, the Harmonic Wave Analyser, and the Acoustic Spectrometer which superseded it. The design, calibration, methods of use, and the interpretation of the results from the latter are described, and a specimen series of readings is given.

The method has been applied to the measurement of the amplitude and frequencies of the harmonic components of complex vibration waves. No claim is made that the pathological processes leading to Raynaud's phenomenon and the bone and joint changes already known to be caused by vibrations depend upon these two physical quantities alone. It is probable that the total energy absorbed by the tissues has also to be considered.

\section{Acknowledgments}

We wish to acknowledge the help and encouragement of Dr. Donald Hunter, Director of the Department, and of Dr. Kenneth Perry. Our thanks are due to Professor D. T. Harris for help and advice, also to Mr. N. Fleming, Head of the Acoustics Section of the National Physical Laboratory, and Mr. B. H. Wallace, late of the Ford Motor Co. Without the very generous assistance of Dr. D. R. Thompson and members of the Vibration Department of the De Havilland Aircraft Co., Ltd., of Messrs. Marconi Instruments, Ltd., who lent the Harmonic Wave Analyser, and of the Controller of the Post Office Research Station, who lent the Acoustic Spectrometer, the work could not have been done.

\section{REFERENCES}

Brailsford, J. F. (1934). Radiology of Bones and Joints, London, p. 27.

Barker, N. W., and Hines, E. A. (1944). Proc. Mayo Clin., 19, 345.

Bennett, G. A., Waine, H., and Bauer, W. (1942). Changes in the Knee Joint at various ages. Commonwealth Fund, New York, p. 68.

Cummins, R. C. (1940). Irish J. med. Sci., 171.

Hunt, J. H. (1936). Quart. J. Med., 19, 399.

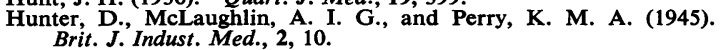

Lindquist, T., and Flemberg, T., (1945). Acta Med. Scand., 121, 309. McLaren, J. W. (1937). Lancet, 2, 1296.

Middleton, E. L. (1930). Ann. Rep. H.M. Chief Insp. Fact., p. 119

Mills, J. H. (1942). Northwest Med., 41, 282.

Seyring, M. (1930). Arch. Gewerbepath. u. Hyg., 1, 359.

Teleky, L. (1927). Quoted from Teleky, L. Occupation and Health Supplement, Geneva, p. 2. Sept. 1938.

Telford, E. D., McCann, M. B., and MacCormack, D. H. (1945) Lancet, 2, 359. 